Issued by Sandia National Laboratories, operated for the United States Department of Energy by Sandia Corporation.

NOTICE: This report was prepared as an account of work sponsored by an agency of the United States Government. Neither the United States Government, nor any agency thereof, nor any of their employees, nor any of their contractors, subcontractors, or their employees, make any warranty, express or implied, or assume any legal liability or responsibility for the accuracy, completeness, or usefulness of any information, apparatus, product, or process disclosed, or represent that its use would not infringe privately owned rights. Reference herein to any specific commercial product, process, or service by trade name, trademark, manufacturer, or otherwise, does not necessarily constitute or imply its endorsement, recommendation, or favoring by the United States Government, any agency thereof, or any of their contractors or subcontractors. The views and opinions expressed herein do not necessarily state or reflect those of the United States Government, any agency thereof, or any of their contractors.

Printed in the United States of America. This report has been reproduced directly from the best available copy.

Available to DOE and DOE contractors from

Office of Scientific and Technical Information

P.O. Box 62

Oak Ridge, TN 37831

Prices available from (703) 605-6000

Web site: http://www.ntis.gov/ordering.htm

Available to the public from

National Technical Information Service

U.S. Department of Commerce

5285 Port Royal Rd

Springfield, VA 22161

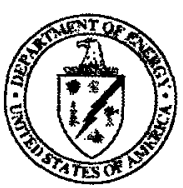




\section{DISCLAIMER}

Portions of this document may be illegible in electronic image products. Images are produced from the best available original document. 
SAND99-2470

Unlimited Release

Printed March 2000

\title{
Construction of File Database Management
}

\author{
Kyle Merrill \\ Materials Aging and Reliability Interfaces \\ Sandia National Laboratories \\ P.O. Box 5800 \\ Albuquerque, NM 87185-0340
}

\begin{abstract}
This work created a database for tracking data analysis files from multiple lab techniques and equipment stored on a central file server. Experimental details appropriate for each file type are pulled from the file header and stored in a searchable database. The database also stores specific location and self-directory structure for each data file. Queries can be run on the database according to file type, sample type or other experimental parameters. The database was constructed in Microsoft Access and Visual Basic was used for extraction of information from the file header.
\end{abstract}




\section{Table of Contents}

\section{Page Numbers}

Problem 1

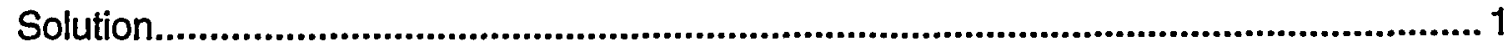

Data Description .......................................................................................................

Software Development.........................................................................................................

Database Management...................................................................................................... 4

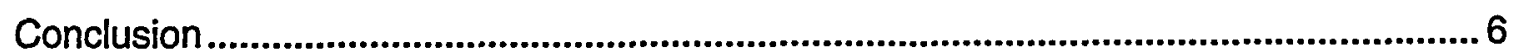

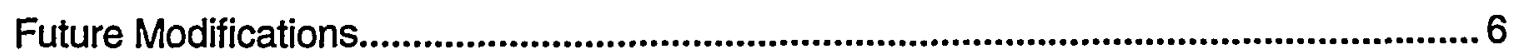

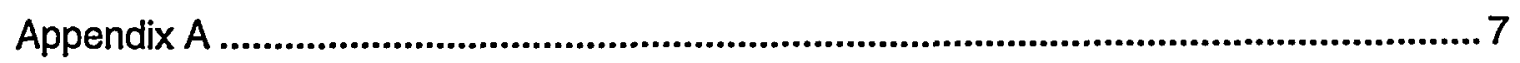

FormGroup frm ...................................................................................................

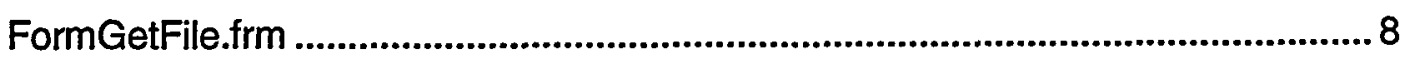

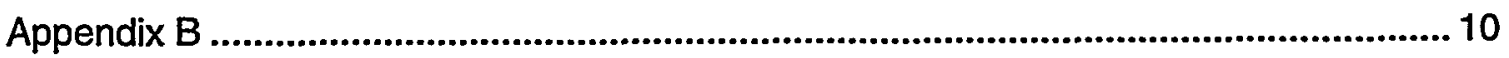

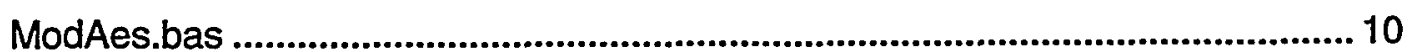

ModPCExplorer.bas ........................................................................................ 12

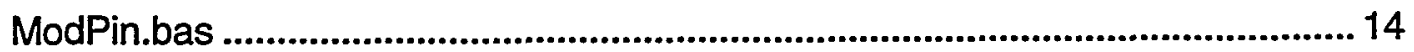

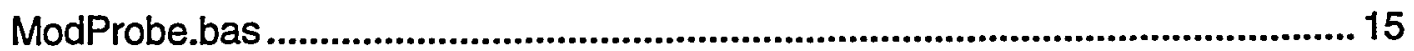

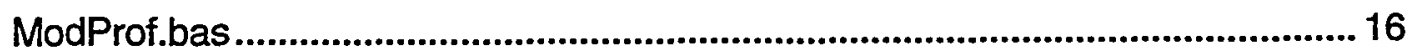

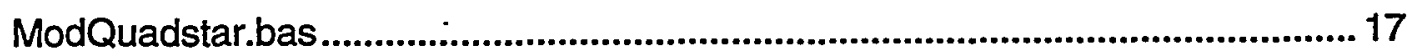

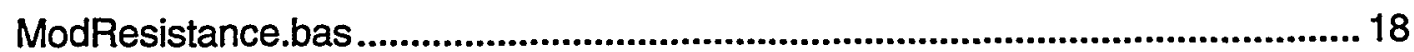

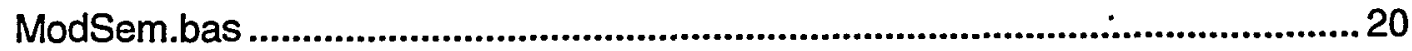

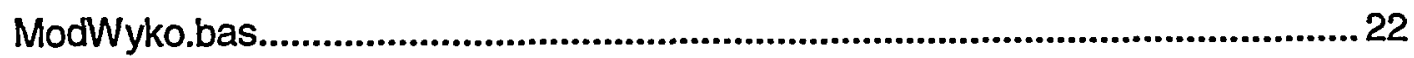

\section{Figures and Tables}

Table 1 Experiment types, perspective File Formats, and Module Filenames ..................2

Fig. 1 First Form seen when Retrieve Data Macro is Run ............................................. 3

Fig. 2 Form seen when any of nine command buttons selected on Fig 1 ...................... 3

Fig. 3 MS Access Import Wizard's Advanced Specifications .......................................... 5

Fig. 4 Macro design time showing the Transfer Text Action and Arguments ................. 5

Fig. 5 FileDateSize Table from the HeaderDataStorage Database .............................. 5 


\section{Title: Construction of File Database Management}

\section{Problem}

There was a need for a database that would keep track of all the files from different lab experiments. This was important because the vast amount of data files would be able to be better managed. One sample would have multiple experiments performed on it and through performing a query the different data files associated with the specific sample could be found.

As the experiments were performed and the data recorded the files were saved on the server. Many experiments were performed with different kinds of software and equipment. This resulted in enormous amounts of data files being saved on the server throughout the years. The problem was trying to remember where the files were, when information was needed on a certain experiment sample. Also those that performed the experiments would like to be able to compare a sample that had different kinds of experiments performed on it. The Find File method, contained within windows, took huge amounts of time to look through the server for files. Also with this method the ability to narrow down the search criteria was limited.

One problem was the programming skills that were needed to accomplish this task were lacking. In counseling with the managers the database developer decided to learn Visual Basic 6.0. This was accomplished through buying some books and taking a one-week course taught by a Microsoft certified teacher. This gave the needed basic skills to start programming source code that would convert the file header into a format that could be imported into the database tables.

\section{Solution}

The header information is extracted from the data files and then recorded in the database. The information in the file header contains the parameters used during the experiment. This will help recall what data file is needed. The user can run a query in the database, searching for the different parameters that tell the user what data file they are looking for.

A program is written to convert the header format into a format that can be imported into the database. This involves different source code in the program, because each set of software and equipment has a different type of file header. The source code needs to read the header information from the data files and then write it to a temporary file in the correct format. The correct format consists of text all on one line separated by semicolons. Some of the files are in ASCll format and some are in Binary format. This requires different techniques of reading the file header from the data file.

This database needs to be easy to use so that one, it will be used, and two, it will save time in finding the needed file information. As this is accomplished, time will be saved and the information obtained from the experiments will be better utilized. The program extracts the needed file header information from the data files so it can be imported into the database. The database is user-friendly which means there is no data entry from the keyboard. This is extremely important, because in order for the database to get used, it has to be efficient and worthwhile to use. 


\section{Data Description}

Microsoft Access was the database that was chosen for this project. It was chosen because it is fairly simple to use and is already on all the computers at Sandia. At the first of the project when trying to import the files directly into the database it was found that the files needed to be in an Access specific format. There were nine different file formats and none of them could be imported directly into Access. In discussing the solution, it was decided a program needed to be written to convert the file header information into a format that could be imported into Access. As Table 1 shows most of the file headers are in ASCII format which made it easy to read the information in the data file headers.

Table 1 Experiment types, perspective File Formats, and Module Filenames

\begin{tabular}{|l|c|c|l|}
\hline Type of Experiment & Header Format & Data Format & Module Filename \\
\hline Resistance & ASCII & ASCII & ModResistance.bas \\
\hline PC Explorer & ASCII & Binary & ModPCExplorer.bas \\
\hline Profilometer & ASCII & ASCII & ModProf.bas \\
\hline Quadstar & ASCII & ASCII & ModQuadstar.bas \\
\hline SEM & ASCII & Binary & ModSem.bas \\
\hline AES & ASCII & Binary & ModAes.bas \\
\hline Wyko & Binary & Binary & ModWyko.bas \\
\hline Pin-on-Disk & ASCII & ASCII & ModPin.bas \\
\hline Probe & ASCII & ASCII & ModProbe.bas \\
\hline
\end{tabular}

The header started at the beginning of the data file for the experiments that had an ASCII header format. This made it easy to read the file information since all that needed to happen was to start reading the file a line at a time, starting at the beginning. The files that had an ASCII header and Binary data were not a problem, because this project dealt with only importing the header information into the database.

The Wyko experiment created more of a problem because both the header and data were in Binary format. Also the header was at the end of the data file. The company that wrote the software was contacted and they provided detailed information about the file and its format. This included what kind of variables the file header contained and how many bytes were associated with each variable. To help know at what byte location the variable from the binary file needed to be read at, a Hex Editor was used, along with the information obtained from the company. The Hex Editor was freeware obtained from the Internet.

\section{Software Development}

There were many different tasks that needed to be accomplished through programming. One of the tasks was to be able to select the correct drive, folder, and file. The first form, (see Fig. 1), had command buttons that when selected source code would load the second form onto the screen. The second form, (see Fig. 2), looked like a file open dialog box. Also there was programming code that calls a specific sub procedure related to the command button selected on the first form. Each sub procedure reads the necessary information from the lab experiment file associated with the sub procedure and then writes the information to temporary files that were created. 


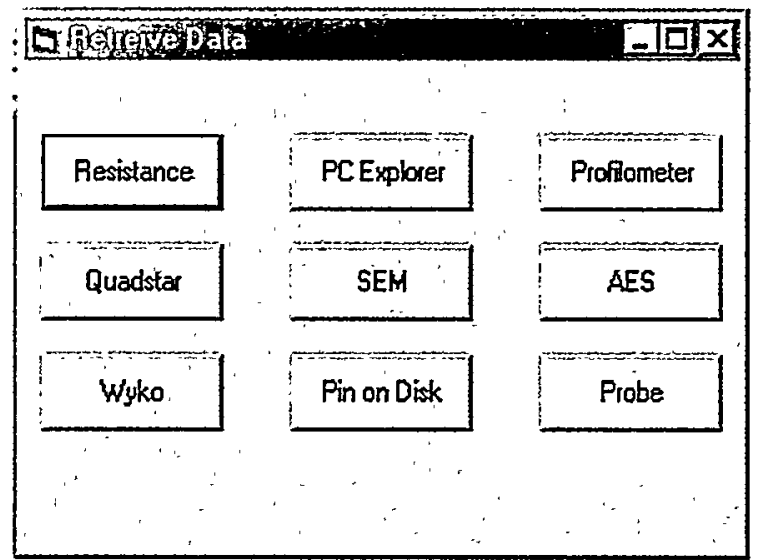

Fig. 1 First Form seen when Retrieve Data Macro is Run

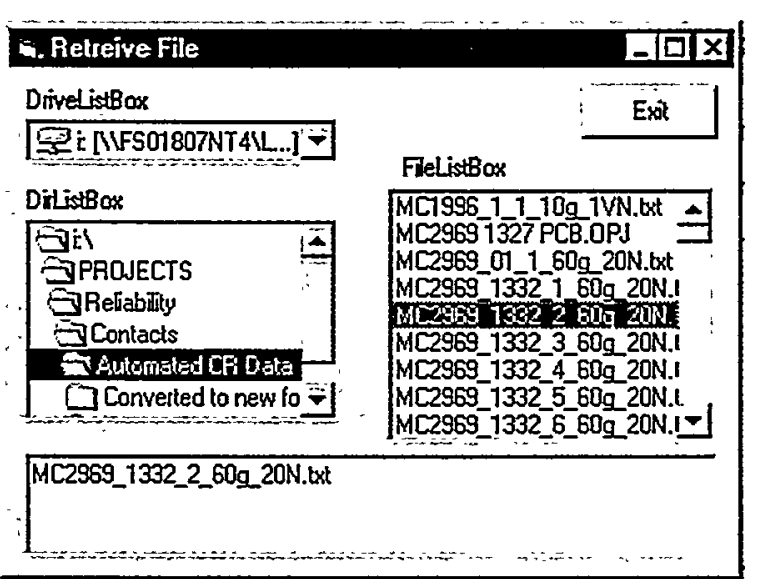

Fig. 2 Form seen when any of nine command buttons selected on Fig 1

The FormGroup.frm, (see Appendix A), was the first code to be executed when the program was run. The nine command buttons, (see Fig. 1), were created into a control array by naming them the same name in the property window of the design form. When this occured each button was assigned an index number starting with zero. The index numbers were used in a Select Case Function, which executes the code under the certain index number corresponding to the command button that was selected. FormGroup.frm also executed the FormGetFile.Show, which let the user see the form in Fig. 2. Also three temporary files were either created or the data in these files was erased when any one of the command buttons was selected.

Next in the program was the FromGetFile.frm code, (see Appendix A), which was the code from the form in Fig. 2. This code let the user navigate through the different drives, directories, folders, and files that were on the computer. Once a file was selected that was when the main part of the code executed. There was an "If Then Elself Function" that calls a certain module if the specific Boolean variable was true. This specific variable was set to true corresponding to the command button selected at the beginning. This code also set up the variables necessary to read and write the data from the data file to the temporary file

All of the modules accomplished the basic goal of reading the data from the correct file and writing the formatted information to the temporary files. Because the data files had differences in the header format there was the need to write code for each type of experiment. To see the actual code written in the modules, refer to Appendix B. In order to read the files that were in ASCll format, the program read the file a line at a time and then extracted the needed information from the line of text. The information was then written to the temporary files. One of the problems experienced was that the file headers were not of the same length. 
ModAes.bas, ModPCExplorer.bas, and ModSem.bas all had extremely similar code because the file headers all were comparable. There were a few differences that required code to be written for each file. The differences were one file would be missing a line or have more lines in the header than the other file type. All of these files had the possibility of having multiple parameters in the header, therefore the code wrote those parameters to the third temporary file. ModSem.bas and ModAes.bas read in the header information differently, but the way the code wrote the information to the temporary file was adjusted so they both could be imported into the same tables in the database.

ModQuadstar.bas and ModResistance.bas were also similar to the previous modules in that they also wrote to three temporary files. The Quadstar data file was separated by tabs instead of spaces so the Split Function had to specify to Split the text that was separated by tabs. The Resistance data file had fourteen lines of text and also had the possibility of having multiple lines after them, depending on the file. The ModResistance.bas code read in the lines of text and then could tell whether or not there were multiple lines after the original fourteen.

The ModPin.bas, ModProbe.bas, and ModProf.bas modules all wrote to only two temporary files. One of the problems experienced by the Pin data was that some of the text lines contained a quotation mark. Access did not like importing this data, even though semicolons separated it, when it had a quotation mark in it. Code was written to replace the quotation mark with a blank space. The Probe data file had two lines of text, which did not have a space after the semicolon while all the other lines had one space. This required two lines of code to be written specifically dealing with those two lines.

In the ModWyko.bas module it was necessary to read the data file as a random access file. With knowledge obtained from the company that produced the Wyko software and a Hex Editor the data file could be read. Source code could be written to read a certain number of bytes for the specific type of variable, pointed at a specific location in the file. Then the binary variables were converted to ASCll a variable at a time, with CStr Function, and then were written to the temporary files.

\section{Database Management}

The program enabled the database user to input all of the data by using the mouse. This accomplished one of the objectives of making the database easy to use by having no keyboard data entry. The source code was created into an executable file, which was then used in a macro in Access by stating the path and filename of the executable. When the Retrieve Data macro ran it called the executable file which started the program. Once a command button on the first form was selected, (Fig.1) another form opens, (Fig. 2) which was similar to an open file form and had an exit button. The file or files to be input into the database could be found by going to the correct directory and folder and then clicking on the filename. The program then read the necessary information from the file and wrote it to the temporary files in the correct format. Now that the temporary files contained the file information in the correct format they could be imported into the Access database. 


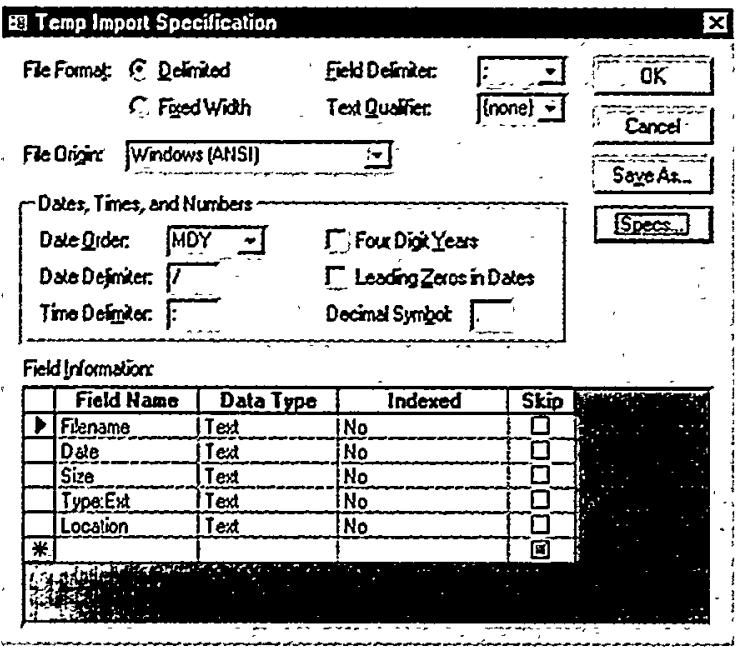

Fig. 3 MS Access Import Wizard's Advanced Specifications

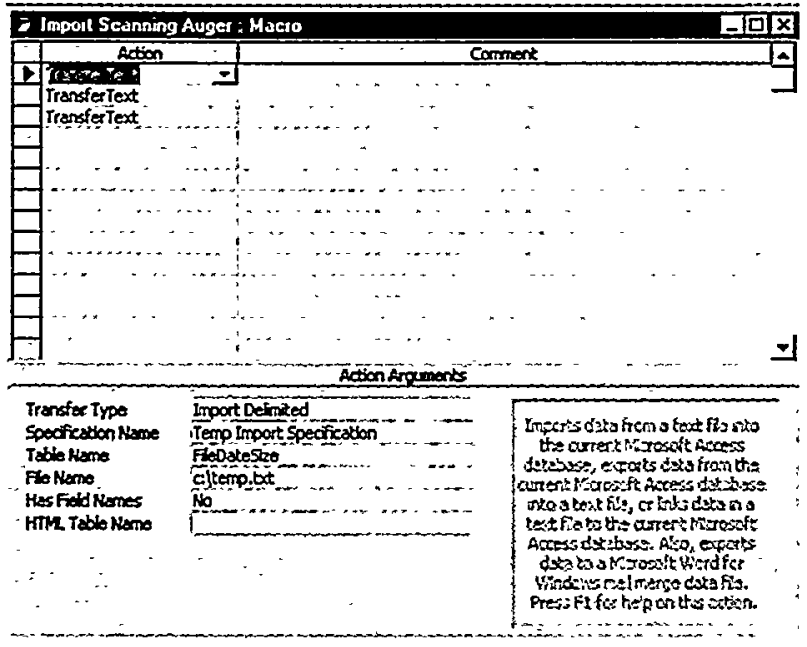

Fig. 4 Macro design time showing the Transfer Text Action and Arguments

Once all the files from a specific experiment had been selected the Exit button could be clicked to leave the program and macro. There were import macros for each different kind of file type and experiment. Indicating the Specification Name, Table Name, and File Name (See Fig. 4) was how the import macro was created. In order to produce the Specification Name the developer had to use Access' import features. Under the file menu was a Get External Data command, and then the Import option was selected. As the developer went through the different steps the computer asked for, there was an advanced button that when selected, the Field Names and Data Type could then be entered in to correspond to the matching table fields (See Fig. 3). This import specification was then saved and the name was placed in the transfer text macro settings corresponding to the correct temporary file and table (See Fig. 4). Under File Name the developer inputed the path and name of the specific temporary file being imported. This needed to correspond to the table entered in the Table Name category where the temporary file data was going to be imported.

\begin{tabular}{|c|c|c|c|c|}
\hline \multicolumn{4}{|c|}{ 瞿 FileDateSize : Table } & $-|\square| x$ \\
\hline & Filename & Date & Type:Ext & Location \\
\hline & 980702 17c rotor, a hi.opd & $6 / 30 / 998: 48: 46$ AM & 449176 opd & $c$ :Idocumentsig80702 17c rotor, a hi.opd \\
\hline & 990403 10.sem & $6 / 8,9911: 30: 51 \mathrm{AM}$ & 104949 sem & $\mathrm{A}: 199040310 . \mathrm{sem}$ \\
\hline & 990405 3.spe & $6 / 8 / 9911: 30: 49 \mathrm{AM}$ & 14109 spe & A: $9904053 . \mathrm{spe}$ \\
\hline & $990407 \mathrm{f}$.dat & $6 / 3019910: 03: 04$ AM & 214137 dat & I:UUSERSUkyleviles for Kylelggo407f dat \\
\hline & a\&b.txt & $6 / 30 / 9910: 07: 42 \mathrm{AM}$ & 544 txt & l:UUSERSUkylelfiles for Kylela\&b.txt \\
\hline & D9122.SPE & $4 / 12 / 9911: 46: 34 \mathrm{AM}$ & 17970 SPE & 1:DATALPSSAp99D9122.SPE \\
\hline & FILEFRM2.ASC & $7 \pi 1999: 05: 47 \mathrm{AM}$ & 2894 & 1:UUSERSUKYleUFILEFRM2ASC \\
\hline 1 & & & & \\
\hline
\end{tabular}

Fig. 5 FileDateSize Table from the HeaderDataStorage Database 
When the specific import macro ran, the information in the temporary files were written to the corresponding tables in the database, resulting in something similar to Fig 5. This particular table contained every data file regardless of its type. The main advantage of this table was the Location field, which helped the user locate a specific file on the server or wherever the file was located. The other tables contained Fields equivalent to the specific experiments data file header. Some of the experiments had one table and some had two, depending on what the data was like in the file header.

\section{Conclusion}

The time to input the data into the database is insignificant compared to the time that will be saved in finding the experiment information that is needed. One can learn how to input the data into the database in minutes. The process is extremely easy and as stated previously requires no keyboard entry.

This project will better utilize the experiments performed on the samples in many ways. One sample will be tested and go through different experiments. By doing a query in the database, the different files associated with the specific sample or with related samples can be found. Large sets of data will be managed and the data can be cross-referenced from multiple analysis. This will result in time saved in trying to find the needed information on the samples tested and will better utilize all the experiments performed.

\section{Future Modifications}

The Visual Basic program is set up in a way that if a new experiment type needs to be added to the database, this can be accomplished with little change to the program. One experiment type is not included in the database because the data file is in a binary format and sufficient information couldn't be obtained from the company that produced the software.

The Quadstar data files also are in binary format and the company does not supply the needed information to read the files. This software has the ability to convert the data and header information into an ASCII file. The ASCII data file can then be read and put into the correct format to be imported into the database. If the knowledge to read these files is obtained through the information that is supplied from these companies then these files in their binary format can also be imported into the database. 


\section{FormGroup.frm}

\section{Appendix A}

'This is the first form that you see. When you click on one of the command buttons

' it opens the Get File form and also assigns the specific boolean variable to true which is ' used in the if then statements

Option Explicit

' Declares variables as Public which means they are shared from form to form

Public blnResistance As Boolean, blnAes As Boolean

Public blnProf As Boolean

Public blnSem As Boolean, blnWyko As Boolean

Public blnPCExplorer As Boolean

Public blnProbe As Boolean, blnPin As Boolean

Public blnQuadstar As Boolean

Public mfso As New FileSystemObject ' 'In using this type of variable one must go to

'Project menu and then References and then

'check Microsoft Scripting Runtime

Private Sub cmdMain_Click(Index As Integer)

' Creates temp files or erases data in them

'A control array which sets the boolean value

' to true depending on which button is selected

End Select

\section{End Sub}

' Creates three temporary files to write the converted header into

Public Sub EraseTempFiles()

Call mfso.CreateTextFile("c:Itemp.txt", True)

Call mfso.CreateTextFile("c:Itemp2.txt", True)

Call mfso.CreateTextFile("c:Itemp3.txt", True)

End Sub 


\section{FormGetFile.frm}

' This form lets you select the specific file that you want from a file open type form Option Explicit

' This correlates to Exit command button and ends the program when clicked

Private Sub cmdExit_Click() End

End Sub

'These next two Sub Procedures makes it so you can select the specific file you want

'from the correct directory and folder

Private Sub dirDirBox_Change()

' Update the file path to the directory path

filFileBox.Path $=$ dirDirBox.Path

End Sub

Private Sub drvDriveBox_Change()

On Error GoTo errorhandler

' Update the directory path to the drive

dirDirBox.Path $=$ drvDriveBox. Drive

Exit Sub

errorhandler:

Dim message As String

' Check for device unavailable error

If Err.Number $=68$ Then

Dim $r$ As Integer

message = "Drive is not available."

$r=$ MsgBox (message, vbRetryCancel + vbCritical, _

"VBHTP: Chapter 14")

' Determine where control should resume

If $r=$ vbRetry Then

Resume

Else ' Cancel was pressed.

Exit Sub

End If

Else

Call MsgBox(Err.Description, vbOKOnly + vbExclamation)

Exit Sub

End If

End Sub

Public Sub filFileBox_Click

Dim theFile As File

Dim mFile As File, mFile2 As File, mFile3 As File 
Dim mTxtStream As TextStream

Dim mts As TextStream, mts2 As TextStream, mts3 As TextStream

' This code assigns the variable of type file to the 3 temp files

'Then assigns the variable of type TextStream to the file for Appending

Set mFile = FormMain.mfso.GetFile("c:Itemp.txt")

Set $m t s=m$ File.OpenAsTextStream(ForAppending)

Set mFile2 = FormMain.mfso.GetFile("c:ltemp2.txt")

Set $\mathrm{mts} 2=\mathrm{mFile2}$.OpenAsTextStream(ForAppending)

Set mFile3 = FormMain.mfso.GetFile("c:Itemp3.txt")

Set $\mathrm{mts} 3=$ mFile3.OpenAsTextStream(ForAppending)

'Assigns the variable of type file to the file that is clicked with mouse

' Then assigns the TextStream variable to Open for Reading from clicked file

Set theFile $=$ FormMain.mfso.GetFile(filFileBox.Path \& "l" \& filFileBox.List(filFileBox.ListIndex))

Set $\mathrm{mTxtStream}=$ theFile.OpenAsTextStream(ForReading)

'This statement writes the information in the parenthesis to the file temp.txt mts.WriteLine (theFile.Name \& ";" \&

theFile.DateCreated \& ";" \&

theFile.Size \& ";" \&

Right\$(theFile.Namē, 3) \& ";" \&

mts. Close

theFile.Path)

txtDisplay. Text $=$ theFile. Name

'These if statements call the correct procedure depending on

' what command button was clicked in the very first form

If FormMain.bInAes = True Then

Call AesData(mTxtStream, mts2, mts3, theFile)

Elself FormMain.bInResistance $=$ True Then

Call ResistanceData(mTxtStream, mts2, mts3, theFile)

Elself FormMain.blnPCExplorer = True Then

Call PCExplorerData(mTxtStream, mts2, mts3, theFile)

Elself FormMain.bInProf $=$ True Then

Call ProfData(mTxtStream, mts2, mts3, theFile)

Elself FormMain.blnSem = True Then

Call SemData(mTxtStream, mts2, mts3, theFile)

Elself FormMain.binWyko = True Then

Call WykoData(mts2, theFile)

Elself FormMain. bInProbe $=$ True Then

Call ProbeData(mTxtStream, mts2, theFile)

Elself FormMain.bInPin $=$ True Then

Call PinData(mTxtStream, mts2, theFile)

Elself FormMain. bInQuadstar $=$ True Then

End If

Call QuadstarData(mTxtStream, mts2, mts3, theFile)

End Sub 


\section{Appendix B}

\section{ModAes.bas}

' Variables in parenthesis are passed to and from the get file form

Public Sub AesData(mTxtStream As TextStream, mts2 As TextStream, _ mts3 As TextStream, theFile As File)

Dim c As String

Dim aa(60) As String, bb() As String

Dim z As Integer, blankposition As Integer

Dim j As Integer, i As Integer

Dim mark(60) As Integer

\section{On Error GoTo ErrorhandlerAes}

' Loops until EOFH is read which is at the end of the Header, blankposition makes

' certain that the array aa(z) has no blank lines

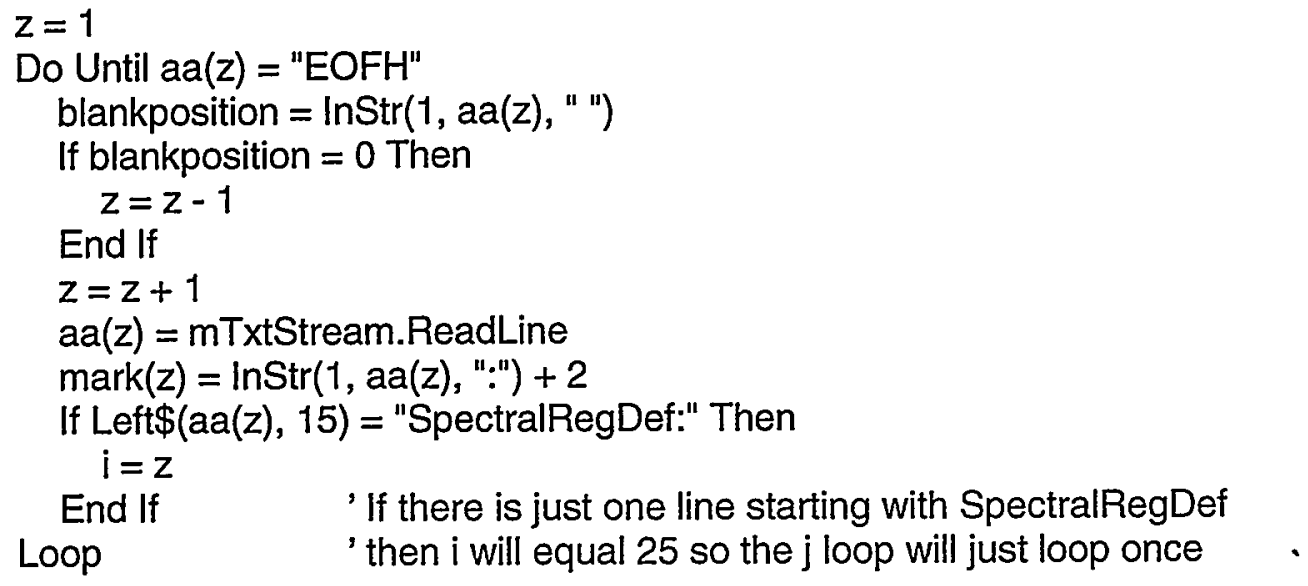

'This loop writes the parameters that may have more than one line to the 3rd temp file

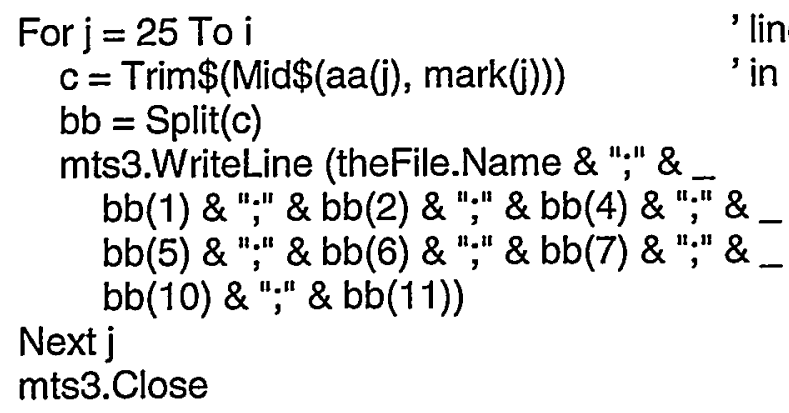

' Trim Function trims any spaces around text stream and Mid Function writes

' the text stream of aa() array starting at position mark()

mts2.WriteLine (theFile. Name \& ";" \&

Trim(Mid(aa(1), mark(1))) \& ";" \& Trim(Mid(aa(2), mark(2))) \& ";" \&

$\operatorname{Trim}(\operatorname{Mid}(\operatorname{aa}(3), \operatorname{mark}(3)))$ \& ";" \& Trim(Mid(aa(4), mark(4))) \& ";" \&

Trim(Mid(aa(5), mark(5))) \& ";" \& "'" \& ";" \& _ 
$\operatorname{Trim}(\operatorname{Mid}(\mathrm{aa}(8), \operatorname{mark}(8)))$ \& ";" \&

Trim(Mid(aa(9), mark(9))) \& ";" \& Trim(Mid(aa(10), mark(10))) \& ";" \&

Trim(Mid(aa(11), mark(11))) \& ";" \& Trim(Mid(aa(12), mark(12))) \& ";" \&

Trim(Mid(aa(13), mark(13))) \& ";" \& Trim(Mid(aa(14), mark(14))) \& ";" \&

Trim(Mid(aa(15), mark(15))) \& ";" \& Trim(Mid(aa(16), mark(16))) \& ";" \& -

Trim(Mid(aa(17), mark(17))) \& ";" \& Trim(Mid(aa(18), mark(18))) \& ";" \&

Trim(Mid(aa(19), $\operatorname{mark}(19)))$ \& ";" \& Trim(Mid(aa(20), mark(20))) \& ";" \& -

Trim(Mid(aa(21), mark(21))) \& ";" \& Trim(Mid(aa(22), mark(22))) \& ";" \& Trim(Mid(aa(23), mark(23))) \& ";" \& Trim(Mid(aa(24), mark(24))) \& ";" \& "I" \& "," \&

Trim(Mid(aa(z - 4), mark(z-4))) \& ";" \&

$\operatorname{Trim}(\operatorname{Mid}(\operatorname{aa}(z-3), \operatorname{mark}(z-3))) \&$ ";" \&

$\operatorname{Trim}(\operatorname{Mid}(a a(z-2), \operatorname{mark}(z-2)))$ \& ";" \&

$\operatorname{Trim}(\operatorname{Mid}(a a(z-1), \operatorname{mark}(z-1))))$

' the aa(z-1to4) writes the four lines mts2.Close

' after the line that may have

'multiple parameters

Exit Sub 'The blank from the "'" five lines up makes this module compatible

ErrorhandlerAes:

' with the Sem module for input into the database

MsgBox "This file cannot be processed please try again.", vbOKOnly, "Bad File"

End Sub 


\section{ModPCExplorer.bas}

'Variables in parenthesis are passed to and from frmGetFile code and this module

Public Sub PCExplorerData(mTxtStream As TextStream, mts2 As TextStream, _ mts3 As TextStream, theFile As File)

Dim c As String

Dim aa(60) As String, bb() As String

Dim z As Integer, blankposition As Integer, i As Integer

Dim j As Integer

Dim mark(60) As Integer

Dim markz3 As Integer, markz2 As Integer, markz1 As Integer

'If an error is experienced at anytime, then this line of code executes On Error GoTo ErrorhandlerPCExplorer

'Loops until text EOFH is read which is at the end of file header

$z=1$

Do Until aa(z) = "EOFH"

blankposition $=\operatorname{InStr}(1$, aa(z), " ")

If blankposition $=0$ Then

$z=z-1$

End If

$z=z+1$

$\operatorname{aa}(z)=m T x t$ Stream.ReadLine

$\operatorname{mark}(z)=\ln \operatorname{Str}(1, \operatorname{aa}(z), ": ")+2$

If Left\$(aa(z), 15) = "SpectralRegDef:" Then

$i=\mathbf{z}$

End If

Loop

'If there are any blank lines $z$ will

' not increment

'Reads one line of text

'Marks position 2 places after :

'Multiple lines may start with this text

'i records last value of $z$ which starts with this text 
Trim(Mid(aa(36), mark(36))) \& ";" \& _

Trim(Mid(aa(37), mark(37))) \& ";" \& _

Trim(Mid(aa(38), mark(38))) \& ";" \& _

Trim(Mid(aa(39), mark(39))) \& ";" \&

Trim(Mid(aa(z - 3), markz3)) \& ";" \& _

$\operatorname{Trim}(\operatorname{Mid}(\mathrm{aa}(\mathrm{z}-2), \operatorname{markz2}))$ \& ";" \&

$\operatorname{Trim}(\operatorname{Mid}(\operatorname{aa}(z-1), \operatorname{mark} z 1)))$

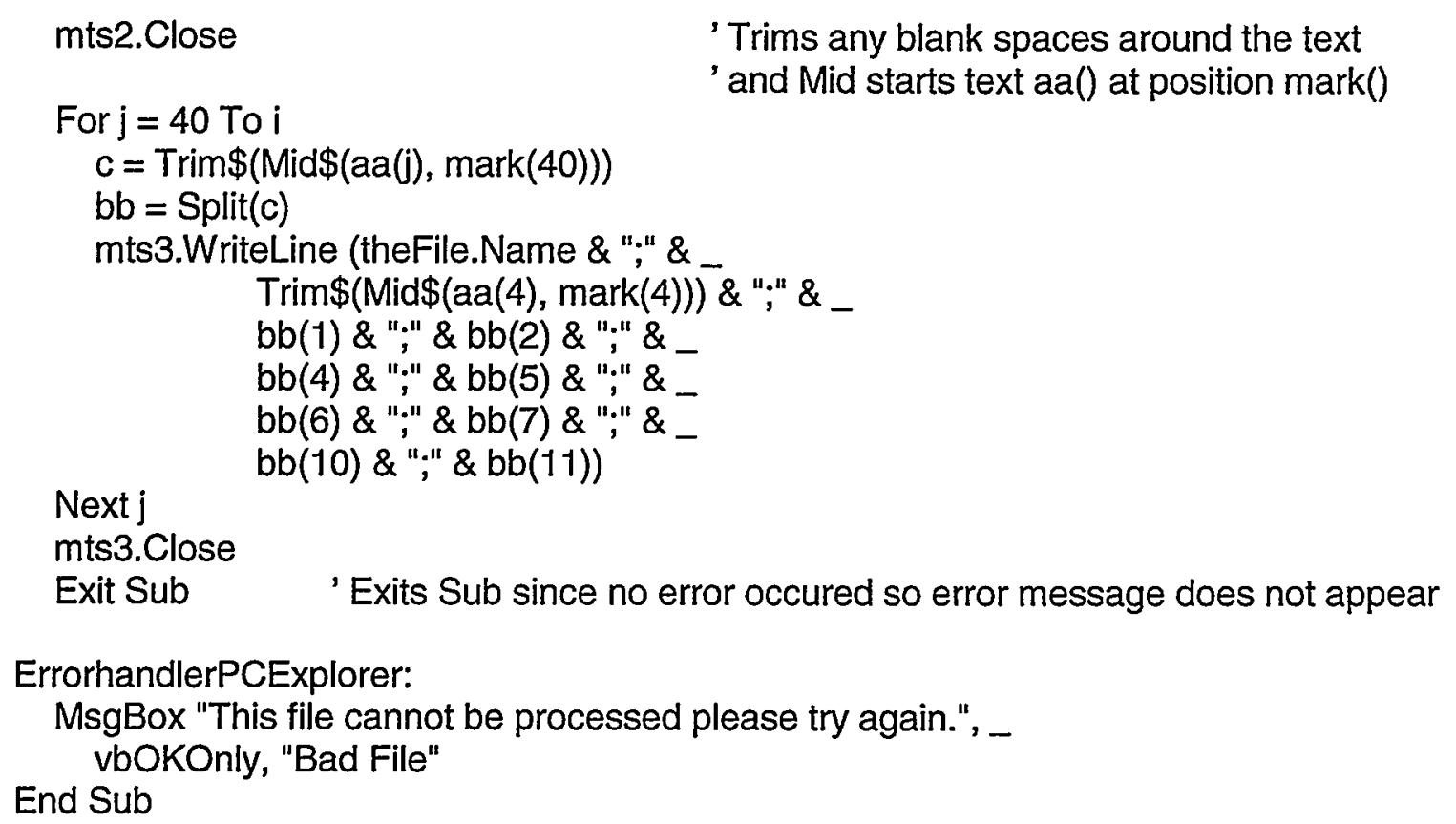




\section{ModPin.bas}

Public Sub PinData(mTxtStream As TextStream, mts2 As TextStream, theFile As File)

Dim aa(60) As String

Dim z As Integer, blankposition As Integer

Dim mark(60) As Integer

Dim position As Integer

Dim Pointer As Integer

Dim Quote As String

On Error GoTo Errorhandler2

$z=1$

Do Until Left(aa(z), 10) = "Processing"

blankposition = $\operatorname{lnStr}(1, \mathrm{aa}(\mathrm{z}), \mathrm{n}$ ")

If blankposition $=0$ Then

$z=z-1$

End If

$z=z+1$

$a a(z)=m T x t S t r e a m$. ReadLine

$\operatorname{mark}(z)=\ln S \operatorname{tr}(1, a a(z), ": ")+2$

For position $=1$ To Len $(a a(z))$

Quote $=\operatorname{Mid}(\operatorname{aa}(\mathrm{z})$, position, 1$)$

Pointer = InStr(position, aa(z), "'"u" )

If Quote $=$ "'mut Then

$\operatorname{Mid}(\mathrm{aa}(\mathrm{z})$, Pointer, 2) $=$ " "

End If

Next position

Loop

'Access had trouble importing text

"because it had a quote "in the line

' this code gets ride of the quote and

' replaces it with a blank space

'Mid(Source text, Starting character position, ' \# of characters to select)

' InStr(Starting search position, string to search,

mark(2) $=\ln S \operatorname{tr}(1, \operatorname{aa}(2), ", ")+2 \quad$ ' what to search for) this returns position in string ' of what searching for

mts2.WriteLine (theFile.Name \& ";" \&

Trim(Mid(aa(2), mark(2))) \& ";" \& -

$\operatorname{Trim}(\operatorname{Mid}(\operatorname{aa}(3), \operatorname{mark}(3)))$ \& ";" \&

Trim(Mid(aa(4), mark(4))) \& ";" \& _

Trim(Mid(aa(5), mark(5))) \& ";" \&

Trim(Mid(aa(6), mark(6))) \& ";" \&

Trim(Mid(aa(7), mark(7))) \& ";" \&

Trim(Mid(aa(8), mark(8))) \& ";" \&

Trim(Mid(aa(9), mark(9))) \& ";" \&

Trim(Mid(aa(10), mark(10))) \& ";" \&

Trim(Mid(aa(13), mark(13))) \& ";" \&

Trim(Mid(aa(14), mark(14))) \& ";" \& _

Trim(Mid(aa(15), mark(15))) \& ";" \& _

$\operatorname{Trim}(\operatorname{Mid}(\mathrm{aa}(16), \operatorname{mark}(16))))$

mts2.Close

Exit Sub

Errorhandler2:

MsgBox "This file cannot be processed please try again.", vbOKOnly, "Bad File" End Sub 


\section{ModProbe.bas}

Public Sub ProbeData(mTxtStream As TextStream, _ mts2 As TextStream, theFile As File)

Dim aa(20) As String

Dim z As Integer

Dim mark(20) As Integer

On Error GoTo ErrorhandlerProbe
For $z=1$ To 14 $a a(z)=m$ TxtStream.ReadLine Next $z$ $\operatorname{mark}(z)=\operatorname{lnStr}(1, a a(z), ": ")+2$

$\operatorname{mark}(10)=\operatorname{lnStr}(1$, aa(10), ":" $)+1$ $\operatorname{mark}(13)=\operatorname{lnStr}(1$, aa(13), ":") +1
'Reads 14 lines

' No space after the semicolon in data file is ' why these lines are needed

'Writes text pulled from theFile onto one line separated by semicolons and puts it into a ' temp file

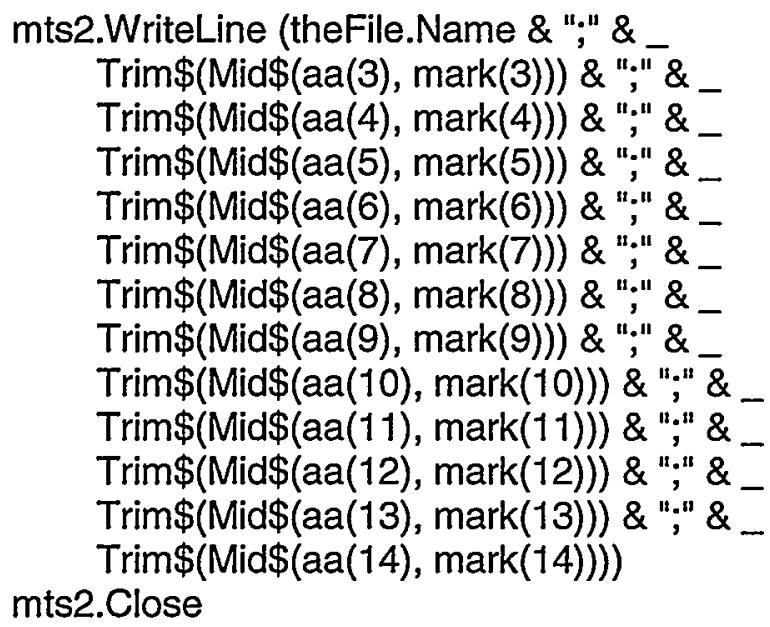

Exit Sub

ErrorhandlerProbe:

MsgBox "This file cannot be processed please try again.", -

End Sub vbOKOnly, "Bad File" 


\section{ModProf.bas}

' Variables are passed from the get file form to be used in this module

' and then they are passed back

Public Sub ProfData(mTxtStream As TextStream, mts2 As TextStream, mts3 As TextStream, theFile As File)

Dim aa(50) As String, bb(50) As String

Dim mark(50) As Integer

Dim z As Integer, blankposition As Integer

On Error GoTo ErrorhandlerProf

For $z=1$ To 45

$\mathrm{aa}(\mathrm{z})=\mathrm{mTxtStream} \cdot$ ReadLine

$\operatorname{mark}(\mathrm{z})=\ln \operatorname{Str}(1, \mathrm{aa}(\mathrm{z}), " \mathrm{\prime \prime})+2$

Next $z$
'Loops 45 times reads text into array aa()

'and marks where colon occurs, then array

' $\mathrm{bb}()$ contains text starting at position mark()

mts2.WriteLine (theFile.Name \& ";" \&

$b b(1)$ \& ";" \& bb(2) \& ";" \&

$b b(3)$ \& ";" \& bb(4) \& ";" \&

$b b(5) \&$ "," \& bb(6) \& ";" \&

$\mathrm{bb}(7) \&$ ";" \& bb(8) \& ";" \&

$b b(9)$ \& ";" \& bb(10) \& ";" \&

$b b(11)$ \& ";" \& bb(12) \& ";" \&

$b b(13)$ \& ";" \& bb(14) \& ";" \&_

$b b(15)$ \& ";" \& bb(16) \& ";" \&

$b b(17)$ \& ";" \& bb(18) \& ";" \& _

$b b(19)$ \& ";" \& bb(20) \& ";" \& _

$b b(21)$ \& ";" \& bb(22) \& ";" \& -

$\mathrm{bb}(23)$ \& ";" \& bb(24) \& ";" \& -

$b b(25)$ \& ";" \& bb(26) \& ";" \& -

$\mathrm{bb}(27)$ \& ";" \& bb(28) \& ";" \& -

$b b(29)$ \& ";" \& bb(30) \& ";" \& -

$b b(31)$ \& ";" \& bb(32) \& ";" \&

$b b(33)$ \& ";" \& bb(34) \& ";" \& -

$b b(35)$ \& ";" \& bb(36) \& ";" \&

$b b(37)$ \& ";" \& bb(38) \& ";" \& _

$b b(39)$ \& ";" \& bb(40) \& ";" \& -

$b b(41)$ \& ";" \& bb(42) \& ";" \& _

$b b(43)$ \& ";" \& bb(44))

mts2.Close

Exit Sub

ErrorhandlerProf:

MsgBox "This file cannot be processed please try again.", vbOKOnly, "Bad File"

End Sub 


\section{ModQuadstar.bas}

Public Sub QuadstarData(mTxtStream As TextStream, _ mts2 As TextStream, mts3 As TextStream, theFile As File)

Dim j As Integer

Dim aa As String, bb() As String

Dim cc1 As String, cc3 As String, cc5 As String

On Error GoTo ErrorhandlerQuadstar

$a \mathrm{a}=\mathrm{m}$ TxtStream.ReadLine

$\mathrm{bb}=$ Split(aa, vbTab)

For $\mathrm{j}=25$ To 101 Step 6

$\mathrm{cc}_{1} 1=\mathrm{bb}(\mathrm{j})$

$\mathrm{cc} 3=b b(j+2)$

$\operatorname{cc} 5=b b(j+4)$

If $\operatorname{cc} 1$ = "Date" Then

Else GoTo Continue

\section{End If}

mts3.WriteLine (theFile.Name \& ";" \& $\operatorname{cc1} \&$ ";" \& $\operatorname{cc3} \&$ ";" \& cc5)

Next $j$

mts3.Close

Continue:

'Trim Function trims line of text starting at position Length of text ' minus length of standard text at beginning of text line

mts2.WriteLine (theFile.Name \& ";" \& bb(0) \& ";" \&

$\operatorname{Trim}(\operatorname{Mid}(b b(3), 1, \operatorname{Len}(b b(3))-7))$ \& ";" \&

$\mathrm{bb}(4)$ \& ";" \& Trim(Mid(bb(9), 1, Len(bb(9)) - 25)) _

\& ";" \& Trim(Mid(bb(12), 1, Len(bb(12)) - 20)) _

\& ";" \& Trim(Mid(bb(15), 1, Len(bb(15)) - 18)) _

\& ";" \& Trim(Mid(bb(18), 1, Len(bb(18)) - 28)) _ .

\& ";" \& Trim(Mid(bb(21), 1, Len(bb(21)) - 13)))

mts2.Close

Exit Sub

ErrorhandlerQuadstar:

MsgBox "This file cannot be processed please try again.", vbOKOnly, "Bad File"

End Sub 


\section{ModResistance.bas}

Public Sub ResistanceData(mTxtStream As TextStream, mts2 As TextStream, _ mts3 As TextStream, theFile As File)

$\operatorname{Dim}$ aa(40) As String, bb() As String

Dim c As String

Dim mark(40) As Integer

Dim blankposition As Integer

Dim z As Integer, i As Integer, j As Integer

Dim Answer As Integer

On Error GoTo Errorhandler2

For $z=1$ To 14

$\mathrm{aa}(\mathrm{z})=\mathrm{mT}$ TtStream.ReadLine

Next $z$
'Reads a line at a time from one to fourteen

' and also marks the position 2 places after:

Do Until Left(aa(z), 4) = "Load"

blankposition = InStr(1, aa(z), "'"')

If blankposition $=0$ Then

'If there is a blank line then $z$ doesn't increment $z=z-1$

End If

$z=z+1$

$\mathrm{aa}(\mathrm{z})=\mathrm{mT}$ tStream.ReadLine

$\operatorname{mark}(z)=\ln \operatorname{str}(1, \operatorname{aa}(z), ": ")+2$

If $z=16$ And $a a(z)=" 1 "$ Then

GoTo Continue

End If

$i=z-1$

Loop

For $j=16$ To $i$

$c=a a(j)$

$\mathrm{bb}=$ Split $(\mathrm{c}, \mathrm{vbTab})$

mts3.Writeline (theFile.Name \& ";" \& $b b(0) \&$ ";" \& bb(1) \& ";" \&

Next j

$b b(2) \& " ; "$; $b b(3))$

' Splits line of text separated by tab

' and puts text in array bb()

' Data file contains no data so skips the

'j For Next Loop

mts3.Close

Continue:

mts2.WriteLine (theFile.Name \& ";" \&

Trim(Mid(aa(1), mark(1))) \& ";" \&

Trim(Mid(aa(2), mark(2))) \& ";" \& -

Trim(Mid(aa(3), mark(3))) \& ";" \& -

Trim(Mid(aa(4), mark(4))) \& ";" \& -

Trim(Mid(aa(5), mark(5))) \& ";" \& -

Trim(Mid(aa(6), mark(6))) \& ";" \& -

Trim(Mid(aa(7), mark(7))) \& ";" \& -

Trim(Mid(aa(8), mark(8))) \& ";" \& _

'Trims text at marked position 
$\operatorname{Trim}(\operatorname{Mid}(\mathrm{aa}(9), \operatorname{mark}(9))) \&$ ";" \&

Trim(Mid(aa(10), mark(10))) \& ";" \&

Trim(Mid(aa(11), mark(11))) \& ";" \&

Trim(Mid(aa(12), mark(12))) \& ";" \&

Trim(Mid(aa(13), $\operatorname{mark}(13)))$ \& ";" \&

Trim(Mid(aa(14), mark(14))))

mts2.Close

Exit Sub

Errorhandler2:

If Err. Number $=62$ Then

Answer = MsgBox("This file contains no data." \& vbCrLf \&

"Do you want to save in Database.", vbYesNo, "Bad File")

If Answer $=$ vbYes Then

Resume Next

Else

Exit Sub

End If

\section{Else}

MsgBox "This file cannot be processed please try again.", vbOKOnly, "Bad File"

End If

End Sub
'If data file contains header only and no data

'Then error message 62 occurs 


\section{ModSem.bas}

' For comments on code see Aes Module

Public Sub SemData(mTxtStream As TextStream, mts2 As TextStream, mts3 As TextStream, theFile As File)

Dim aa(60) As String, bb() As String, c As String

Dim z As Integer, blankposition As Integer, i As Integer

Dim j As Integer

Dim mark(60) As Integer

\section{On Error GoTo Errorhandler2}

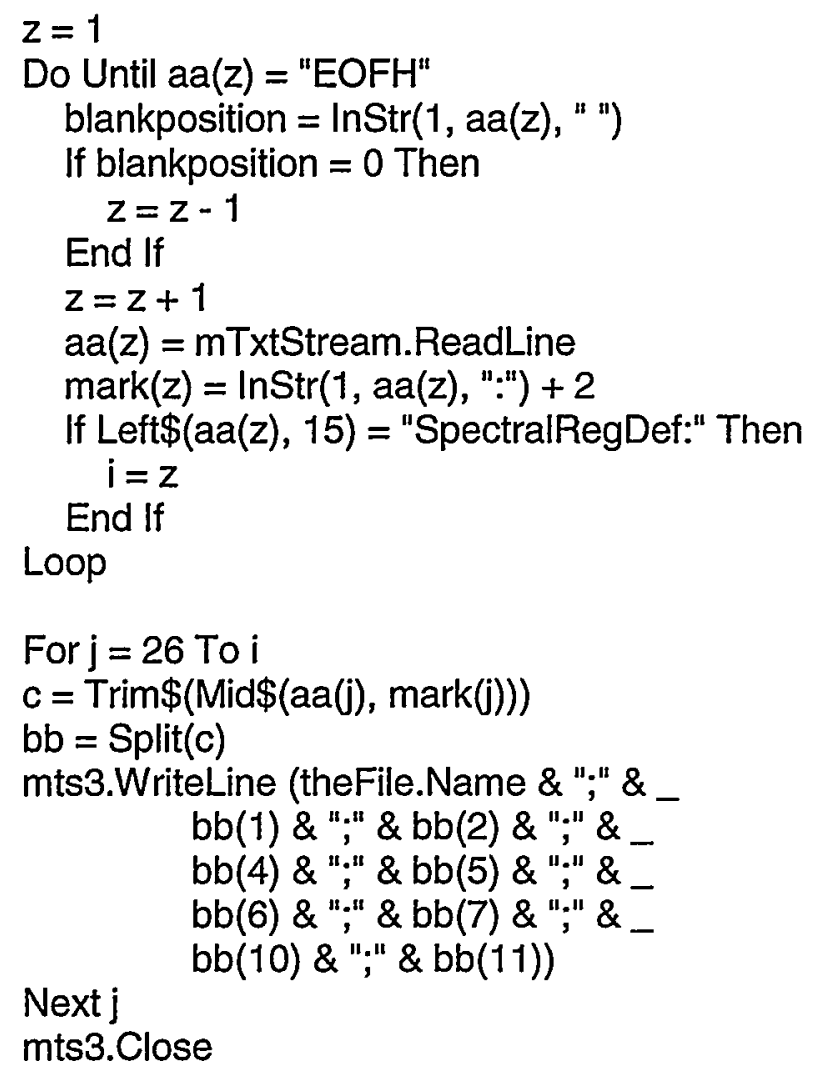

mts2.WriteLine (theFile.Name \& ";" \&

$\operatorname{Trim}(\operatorname{Mid}(\operatorname{aa}(1), \operatorname{mark}(1))) \&$ ";" \& Trim(Mid(aa(2), $\operatorname{mark}(2)))$ \& ";" \&

Trim(Mid(aa(3), mark(3))) \& ";" \& Trim(Mid(aa(4), mark(4))) \& ";" \& _

Trim(Mid(aa(5), mark(5))) \& ";" \& Trim(Mid(aa(8), mark(8))) \& ";" \& _

Trim(Mid(aa(9), $\operatorname{mark}(9)))$ \& ";" \& Trim(Mid(aa(10), mark(10))) \& ";" \&

Trim(Mid(aa(11), mark(11))) \& ";" \& Trim(Mid(aa(12), mark(12))) \& ";" \&

Trim(Mid(aa(13), mark(13))) \& ";" \& Trim(Mid(aa(14), mark(14))) \& ";" \& _

Trim(Mid(aa(15), $\operatorname{mark}(15)))$ \& ";" \& Trim(Mid(aa(16), mark(16))) \& ";" \& _

$\operatorname{Trim}(\operatorname{Mid}(\operatorname{aa}(17), \operatorname{mark}(17)))$ \& ";" \& Trim(Mid(aa(18), mark(18))) \& ";" \& _

Trim(Mid(aa(19), mark(19))) \& ";" \& Trim(Mid(aa(20), mark(20))) \& ";" \& -

Trim(Mid(aa(21), mark(21))) \& ";" \& Trim(Mid(aa(22), mark(22))) \& ";" \& _

Trim(Mid(aa(23), mark(23))) \& ";" \& Trim(Mid(aa(24), mark(24))) \& ";" \&

Trim(Mid(aa(25), mark(25))) \& ";" \& _ 
$\operatorname{Trim}(\operatorname{Mid}(\operatorname{aa}(z-5), \operatorname{mark}(z-5))) \&$ ";" \&

Trim(Mid(aa(z - 4), mark(z-4))) \& "", \&

$\operatorname{Trim}(\operatorname{Mid}(\operatorname{aa}(z-3), \operatorname{mark}(z-3)))$ \& ";"; \&

$\operatorname{Trim}(\operatorname{Mid}(\operatorname{aa}(z-2), \operatorname{mark}(z-2)))$ \& ";" \&

$\operatorname{Trim}(\operatorname{Mid}(\operatorname{aa}(z-1), \operatorname{mark}(z-1))))$

mts2.Close

\section{Exit Sub}

Errorhandler2:

MisgBox "This file cannot be processed please try again.", vbOKOniy, "Bad File"

\section{End Sub}




\section{ModWyko.bas}

Public Sub WykoData(mts2 As TextStream, theFile As File)

Dim strTime As String * 9

Dim strDate As String * 9

Dim OffsetDac As Single

Dim SpareADC As Single

Dim MeasMode As String * 10

Dim Vibration As Single

Dim Pixel_size As Single

Dim Measslope As Single

Dim Magnification As Single

Dim Wavelength As Single

Dim GlobalOriginX As Single, GlobalOriginY As Single

Dim StageX As Single, StageY As Single

Dim Pupil As Single

Dim TiltX As Single, TiltY As Single

Dim Use_XYR_cent As Integer

Dim Use_XYR_spac As Integer

Dim Pupil_diam As Single

Dim XYR_x_spac As Single

Dim Terms_String As String * 40

Dim Data_Restore As String * 10

Dim Data_Invert As String * 10

Dim Filt_Type As String * 40

Dim Vol_opt_String As String * 40

Dim UseApodization As Integer

Dim Aspect As Single

Dim Title As String * 20

Dim Note As String * 60

On Error GoTo ErrorhandlerWyko

Open theFile For Binary Access Read As \#1 Get \#1, 447614, strTime

Open theFile For Binary Access Read As \#2 Get \#2, 447624, strDate

Open theFile For Binary Access Read As \#3

Get \#3, 447633, OffsetDac

Open theFile For Binary Access Read As \#4

Get \#4, 447637, SpareADC

Open theFile For Binary Access Read As \#5

Get \#5, 447641, MeasMode

Open theFile For Binary Access Read As \#6

Get \#6, 447651, Vibration

Open theFile For Binary Access Read As \#7

Get \#7, 447655, Pixel_size

Open theFile For Binary Access Read As \#8

Get \#8, 447659, MeasSlope

Open theFile For Binary Access Read As \#9

Get \#9, 447663, Wavelength
'Reads specific number of bytes

' at position indicated, number of

' bytes corresponds to the variable 
Open theFile For Binary Access Read As \#10 Get \#10, 447667, Magnification

Open theFile For Binary Access Read As \#11 Get \#11, 447671, GlobalOriginX

Open theFile For Binary Access Read As \#12 Get \#12, 447675, GlobalOriginY

Open theFile For Binary Access Read As \#13 Get \#13, 447679, StageX

Open theFile For Binary Access Read As \#14 Get \#14, 447683, StageY

Open theFile For Binary Access Read As \#15 Get \#15, 448887, Pupil

Open theFile For Binary Access Read As \#16 Get \#16, 448891, TiltX

Open theFile For Binary Access Read As \#17 Get \#17, 448895, TiltY

Open theFile For Binary Access Read As \#18 Get \#18, 448899, Use_XYR_cent

Open theFile For Binary Access Read As \#19 Get \#19, 448901, Use_XYR_spac

Open theFile For Binary Access Read As \#20 Get \#20, 448903, Pupil_diam

Open theFile For Binary Access Read As \#21 Get \#21, 448907, XYR_x_spac

Open theFile For Binary Access Read As \#22 Get \#22, 448911, Terms_String Open theFile For Binary Access Read As \#23 Get \#23, 448951, Data_Restore

Open theFile For Binary Access Read As \#24 Get \#24, 448961, Data_Invert

Open theFile For Binary Access Read As \#25 Get \#25, 448971, Filt_Type

Open theFile For Binary Access Read As \#26 Get \#26, 449011, Vol_opt_String

Open theFile For Binary Access Read As \#27 Get \#27, 449051, UseApodization

Open theFile For Binary Access Read As \#31 Get \#31, 449053, MaskingString Open theFile For Binary Access Read As \#28 Get \#28, 449093, Aspect

Open theFile For Binary Access Read As \#29 Get \#29, 449097, Title

Open theFile For Binary Access Read As \#30 Get \#30, 449117, Note

' CStr converts the variable in parenthesis to String variable

mts2.WriteLine (theFile.Name \& ";" \& Trim(strDate) \& ";" \&

Trim(Title) \& ";" \&

Trim(Note) \& ";" \& CStr(OffsetDac) \& ";" \&

CStr(SpareADC) \& ";" \& Trim(MeasMode) \& ";" \& _ CStr(Vibration) \& ";" \& CStr(Pixel_size) \& 
";" \& CStr(MeasSlope) \& ";" \&

CStr(Wavelength) \& ";" \& CStr(Magnification) \& ";" \&

CStr(GlobalOriginX) \& ";" \& CStr(GlobalOriginY) \& ";" \&

CStr(StageX) \& ";" \& CStr(StageY) \& ";" \&

CStr(Pupil) \& ";" \& CStr(TiltX) \& ";" \&

CStr(TiltY) \& ";" \& CStr(Use_XYR_cent) \& ";" \&

CStr(Use_XYR_spac) \& ";" \& CStr(Pupil_diam) \& ";" \&

CStr(XYR_X_spac) \& ";" \& Trim(Terms_String) \& ";" \& _

Trim(Data_Restore) \& ";" \& Trim(Data_Invert) \& ";" \&_

Trim(Filt_Type) \& ";" \&

Trim(Vol_opt_String) \& $\overline{\text { ";" }}$ \&

CStr(UseApodization) \& ";" \&

Trim(MaskingString) \& ";" \& _

CStr(Aspect))

mts2.Close

Exit Sub

ErrorhandlerWyko:

MsgBox "This file cannot be processed please try again.", vbOKOnly, "Bad File"

End Sub 
Distribution:

$1 \quad$ MS 0340

$1 \quad$ MS 0340

$1 \quad$ MS 0340

1 MS 0340

1 MS 0340

$1 \quad$ MS 0340

1 MS 0340

1 MS 9018

2 MS 0899

$1 \quad$ MS 0612
Wendy Cieslak, 1832

Michael Dugger, 1832

William McNamara, 1832

James Ohlhausen, 1832

Diane Peebles, 1832

Greg Poulter, 1832

Elizabeth Sorroche, 1832

Central Technical Files, 8940-2

Technical Library, 4916

Review \& Approval Desk, 4912

For DOE/OSTI 